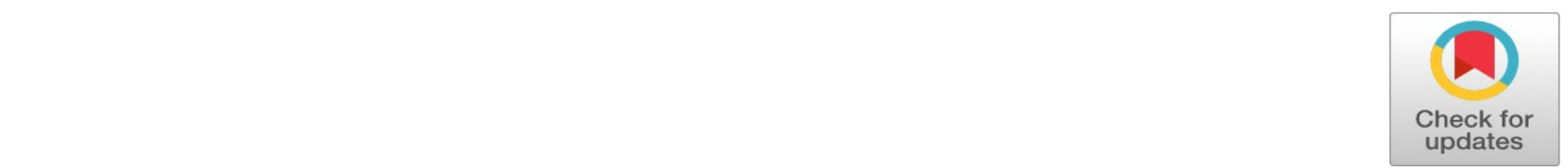

PRIMARY RESEARCH

\title{
Sequence behavior of vortical development and evolution of a low-speed rectangular jet
}

\author{
Yung-Lan Yeh ${ }^{1}$, Chih-Huang Chiang ${ }^{2^{*}}$ \\ ${ }^{1}$ China University of Science and Technology, Taipei, Tiwan \\ ${ }^{2}$ Air Force Institute of Technology, Ohio, $\mathrm{OH}$
}

\begin{abstract}
Index Terms
vortical Structure

Rectangular Jet

Smoke Flow

Abstract - The main objective of present study is to understand the vortical structure development and evolution of a low-speed rectangular jet via smoke flow visualization. The side- and front-view near the jet exit are utilized simultaneously to know the development relationship in detail. Results not only confirm the development of vortex ring but also indicate this process has strong relationship with the vortical structure development in a plane jet.
\end{abstract}

Received: 29 July 2017

Accepted: 31 July 2017

Published: 21 August 2017

(C) 2017 The Author(s). Published by TAF Publishing.

\section{INTRODUCTION}

Turbulence is one of the most challenging fields of fluid mechanics. There had been extensive researches and discussions focused on the shear flow related to the growing and evolution of turbulent flows. The instability of the shear flow may bring about complex phenomena even though the initial and boundary conditions are very simple. This property promotes significant mass and momentum transports between different regions of the flow fields. Related applications include the fuel-gas mixing, the noise reduction, and the high lift devices. The flow field is divided into the shear-layer region and the potential core region (where the stream-wise velocity distributions remain flat). The transport and mixing of mass and momentum in jet flows are closely related to the vortical structures which are originated from the instabilities of the free shear layer. With experimental results in jet and mixing layer, Crow \& Champagne [1], Brown \& Roshko [2], and Browand \& Weidman [3] proved that the large-scale coherent structures were the intrinsic features in free shear flow. At the early stage of the development of a free shear flow, Kelvin-Helmholtz instability is induced due to the presence of the velocity gradient. Through the pairing and the merging process, the vortices form larger structures [4]. Such an interacting process provided the primary mechanism for the mixing and the flow entrainment of free shear flow. The evolution of coherent structures is closely related to the propagation and interaction of the instability waves. Based on the subharmonic evolution model proposed by Ho [5, 6], the occurrence of vortex merging is a result of the evolution of the subharmonic instabilities. The interaction of two rows of vortices is more significant near the end of potential core, and the preferred mode dominates the following structures [7]. In order to analyze the flow structure development in a simple way, most researchers adopt nozzle with high aspect ratio. Typically, the value of aspect ratio is larger than 20 and this kind of nozzle is also called the plane jet. Then the phenomena of roll-up, merging, and pairing of vortex can be visualized and analyzed easily. But when the aspect ratio is lower than 20 , also called the rectangular jet, the vortical development in the corner will connect each other to form the vortex ring

\footnotetext{
${ }^{*}$ Corresponding author: Chih-Huang Chiang

†Email: ylyeh1229@gmail.com
} 
which has 3D characteristics. In view of these facts, there is few related reference investigating the $2 \mathrm{D}$ and $3 \mathrm{D}$ flow structures at the mean time. Present work focuses on the development and relationship of 2D and 3D flow structures using advanced smoke flow visualization method. The side and front views of flow patterns at different stream-wise locations are examined simultaneously. Beside above study topic, the transformation relationship is another important issue to be further studied here. The so-called transformation relationship is the relation between 3D vortex ring and 2D flow structures development. There exist two different development ways. The vortex ring is mainly caused by secondary instability flow in the corner. But the 2D flow structure is induced by the Kelvin-Helmholtz instability.

\section{EXPERIMENTAL FACILITIES AND SETUP}

The low-speed wind tunnel and advanced smoke flow visualization method are two main experimental facilities in this study. In order to confirm the accuracy of experiment, all facilities are well-adjusted. Their detail setup will be mentioned as following.

\section{A. Low-Speed Wind Tunnel}

The experiments were performed in a low-speed airjet tunnel (as shown in Figure 1).

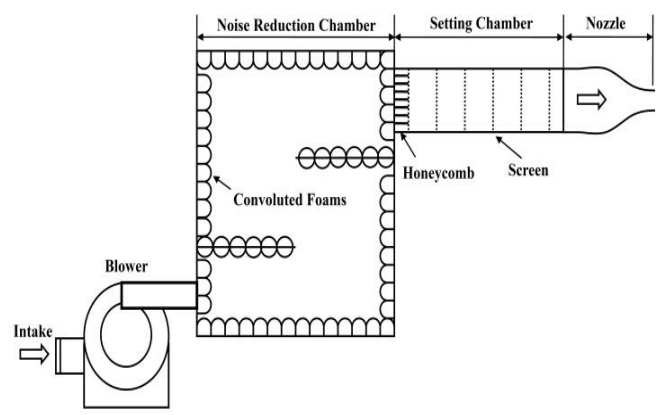

Fig. 1. Sketch of the low-speed wind tunnel

Air flow is generated by a centrifugal blower. The blower noise is reduced by an inlet muffler and a noise reduction chamber. The settling chamber consists of one honeycomb and six porous screens for further improving the flow quality. A nozzle of fifth-order-polynomial profile with area contraction ratio of 20 connects to the outlet of settling chamber to produce the desired jet flow. The noz zle exit is $40 \mathrm{~mm}$ high and $100 \mathrm{~mm}$ wide and the corresponding aspect ratio is 2.5 . The operational exit velocity ranges from 1 to $25 \mathrm{~m} / \mathrm{s}$. The turbulence intensity at the centre of the jet exit is no more than $0.8 \%$. A Pitot probe of $1 \mathrm{~mm}$ in diameter was placed at the jet exit to measure the exit velocity for reference velocity. The Pitot probe was attached to a differential pressure transducer whose precision was $\pm 0.005 \mathrm{~mm} \mathrm{H}_{2} \mathrm{O}$.

\section{B. Advanced Smoke Visualization Method}

Smoke-flow method is an easy way to see the flow pattern by eyes or camera. The smoke is mainly produced by a smoke generator and then is taken into flow field by working fluid. In previous application, the jet flow was visualized and analyzed by smoke-flow method $[8,9]$. The flow pattern and topology of vortices were investigated in detail. Based on previous experimental experiences, smoke flow visualization method had two major disadvantages. The first, the flow field must fill with smoke when flow pattern was visualized in the past. The smoke requirement must increase for aerodynamic analysis in large-scale suction type wind tunnel. It is hard to visualize flow structure under the condition of filled with smoke. The second, the traditional smoke flow method cannot be used to observe local flow field.

Therefore, an advanced smoke flow visualization method is necessary to be developed. Yeh [9] developed a high-pressure smoke flow visualization system from old type smoke flow method and had been improved to visualize local flow structure. Advanced smoke visualization system is composed by concentration box made by wood and a smoke generator. High-pressure flow produced by a compressor was imported into concentration box and then brought out smoke. The largest amount of smoke production reaches 18,000 cubic feet per minute. The odorless, tasteless, and high concentrations of water-based smoke oil was used and stored in a 5-liter oil tank. A tapered device (funnel-like) is equipped at exit end inside tank to avoid pressure drop due to compressor. The output smoke flow approaches laminar flow after tuning by regulator. So, the true flow condition can be faithfully performed by streak line and reveal qualitative phenomena. A settling chamber was set up to stabilize pressure and collect condensate water. Amount of smoke, endurance or time interval are controlled by a remote controller. Detail design and construction are shown in Figure 2. 


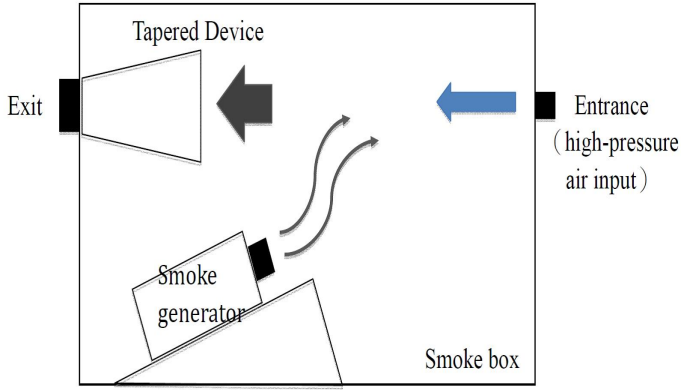

Fig. 2 . Detailed design and construction of smoke box [9]

After preliminary experiments [9], that device was confirmed experimentally and had proved the ability to visualize local flow property at various different Reynolds number. It has very high convenience and adaptability in use. It is also easy to adjust for different type or velocity of flow field. It can enhance the qualitative researches about fluidand aero-dynamics effectively.

\section{Lighting and Image Recording System}

Light source is a semi-conductor laser with $532 \mathrm{~nm}$ wavelength. The max operation power is $1.2 \mathrm{~W}$. In order to narrow laser beam and enhance concentration power, laser light is first emitted through two different focus length lenses to perform collimating. Finally pass through the cylindrical lens and form a high power laser sheet. The spread angle is about $20^{\circ}$. Image recording system is a digital high-speed camera made by SONY Company. Image resolution is fix in $1240 \times 1024$ pixel and frame speed is 5,000 frames per second.

\section{RESULTS AND DISCUSSION}

The Cartesian coordinate is used in present work and the coordinate at nozzle exit is shown in Figure 3.

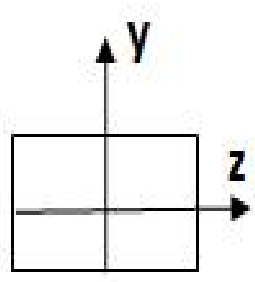

(a)

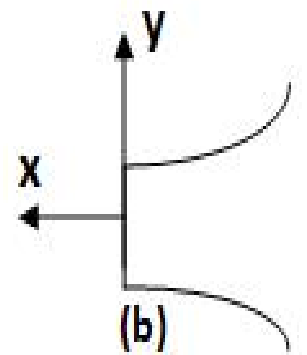

(b)

Fig. 3 . The cartesian coordinates at jet exit (a) front-view; (b) side-view 


\section{B. Development of the Vortex Ring}

Firstly, the uniform core of jet exit is shown in Figure 5(a). It means that the used jet has a good performance under operational speed. Because of small aspect ratio, the vortical structure due to secondary instability in the shear layer at four nozzle corners will develop, grow, and connect each other to form the vortex ring finally. In the early stage of vortical development, the individual vortices at four corners grow slightly (see Figure 5(b)). The main development mechanism is the secondary instability due to corner.

As the growth of vortex, the area of central core becomes small because the vortices in the corner become larger and distorted (as shown is Figure 5(c)). At following stream-wise location, the front view of jet becomes much more chaos (as shown in Figure 5(d)). That is because the vortical interaction of four corners, which makes flow, becomes turbulent. The bigger size of vortices will meet and merge together. For a natural jet, the vortex after pairing and merging processes will be easy to breakdown and then become small-scale turbulence. In Figure 5(e), the appearance of jet view is not like the original one. It likes turbulence more. The stream-wise location here is after the end of potential core and the vortices had been broken down already at this location.

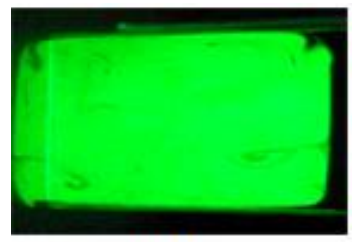

(a)

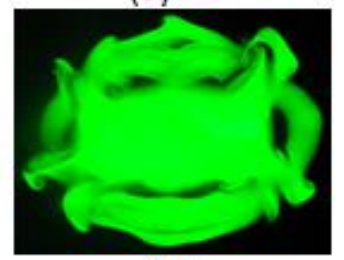

(c)

(e)

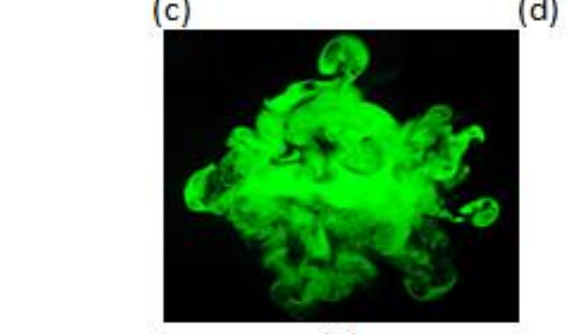

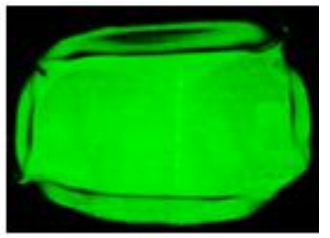

(b)

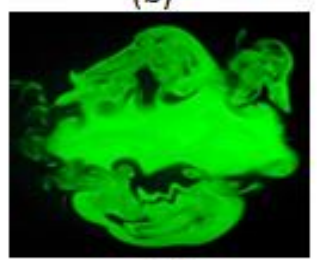

(d)
Fig. 5 . Front view of vortex ring $\mathrm{x} / \mathrm{H}=$ (a) 0.5; (b) 1.5; (c) 2.5; (d) 3.5; (e) 4

\section{Development and Transformation Relationship}

There were some excellent researches related to jet flow. $[3,11,12]$ indicate that the jet mixing and spreading properties will be enhanced due to the growth of vortical structure. If the flow field is excited at its fundamental frequency, the mixing properties in the upstream will further increase $[13,14,15,16,17]$. However, if the jet flow is excited at relative low frequency, two order at least, the flow structure after the end of potential core will be much more organized and the mixing properties will be enhanced significantly [10]. During the vortical development process, the area of potential core, where the speed inside is maintained constant, will decrease simultaneously. According to the arguments mentioned above, the mixing is related to the vortical development and growth, especially the vortical scale. As the scale of vortex becomes larger, the appearance of vortex will change in width and height. At this time, the 2D vortex, which develops in the difference side, will meet each other and start to form 3D structure if the aspect ratio of jet nozzle is small. That is why the vortical structures connect each other and start to form the vortex ring at the position where the $2 \mathrm{D}$ vortex grows large enough. But after the end of potential core of natural jet, the 3D vortical structure is formed and becomes much more chaos without any excitation. Therefore, there is not any obvious coherent structure in front view of jet flow. The whole flow structures of corresponding stream-wise locations of side- and front-views are shown in Figure 6.
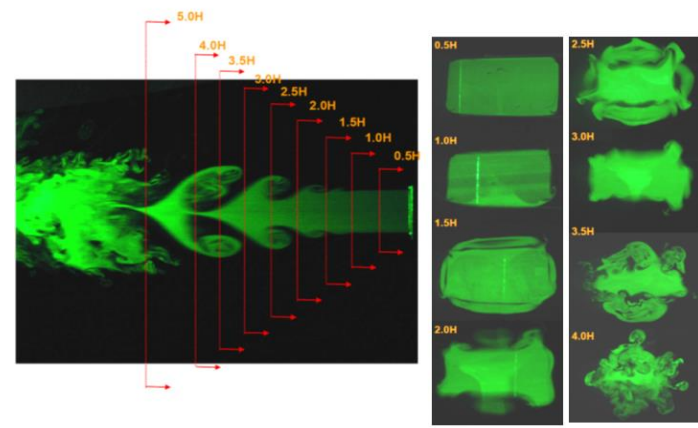

Fig. 6. Whole flow structures of corresponding stream-wise locations of side- and front-views

Basically, the development of vortex ring is correspondent to that of 2D flow structure. Because of velocity gradient due to the Kelvin-Helmholtz instability, the 2D flow structure develops in the shear layer. After absorbing energy from mean flow, the vortical structure then grows and rolls up. But near the corner, the secondary instability flow 
will be induced and then combine 2D flow structures to form the vortex ring. In the downstream, the 2D flow structure breaks down to small-scale eddy and the vortex ring disappears simultaneously. Their developments are closely related.

\section{CONCLUSION}

Present work focuses on the development and relationship of 2D and 3D flow structures via advanced smoke flow visualization method. The side and front views of flow patterns at different stream-wise locations are examined simultaneously. And the transformation relationship is another important issue to be further studied here. The development of vortex ring is correspondent to that of 2D flow structure. Because of velocity gradient due to the Kelvin-Helmholtz instability, the 2D flow structure develops in the shear layer. After absorbing energy from mean flow, the vortical structure rolls up and grows in size. At the corner of jet nozzle, the secondary instability flow is induced and then combines

2D flow structures to form the vortex ring. In the downstream, the 2D flow structure breaks down to small-scale eddy and the vortex ring disappears simultaneously. The whole flow structures become turbulent finally. The main contribution of this study is to study the development and relationship between 2D and 3D flow structures in detail. The advanced smoke flow visualization method is the main experimental equipment adopted here. The transformation and development process and each other's connection are two main arguments.

\section{Acknowledgement}

Ministry of Science and Technology of R.O.C. supports this work with contract number of MOST 105-2221-E-157-005-MY2.

\section{REFERENCES}

[1] S. C. Crow and F. H. Champagne, "Orderly structure in jet turbulence," Journal of Fluid Mechanics, vol. 48, no. 10, pp. 547-591, 1970.

DOI: $10.1017 / \mathrm{S} 0022112071001745$

[2] F. K. Brown and A. Roshko, "On the density effects and large structure in turbulent mixing layers," Journal of Fluid Mechanics, vol. 64, no. 4, pp. 775-816, 1974.

DOI: $10.1017 / \mathrm{S} 002211207400190 \mathrm{X}$ 
[14] Y. C. Chao and J. M. Han, "The evolution mechanism of the controlled flow structure of a round jet under acoustic excitation," in Proceedings of Shear Flow Conference, AIAA-89-0922, Tempe, AZ, 1989.

DOI: https://doi.org/10.2514/6.1989-992

[15] H. Fiedler and P. Mensing, "The plane turbulent shear layer with periodic excitation," Journal of Fluid Mechanics, vol. 150, pp. 281-309, 1985.

DOI: $10.1017 / \mathrm{S} 0022112085000131$
[16] A. K. M. F. Hussain, "Coherent structures and studies of perturbed and unperturbed jets," in Proceedings of The Role of Coherent Structures in Modelling Turbulence and Mixing Springer, Berlin, Germany, 1981.

DOI: https://doi.org/10.1007/3-540-10289-2_30

[17] C. M. Ho, "Local and global dynamics of free shear layers," in Proceeding of the Symposium on Numerical and Physical Aspects of Aerodynamic Flow, Long Beach, CA, 1982.

— This article does not have any appendix. — 